\title{
Human Systems Integration Design: Which Generalized Rationale?
}

\author{
Romain Lieber ${ }^{2, *}$ and Didier Fass ${ }^{1,2}$ \\ ${ }^{1}$ ICN Business School, Artem Augmented Human project \\ ${ }^{2}$ Mosel team, LORIA, University of Lorraine, \\ Campus Scientifique - BP 239, France \\ didier.fass@loria.fr
}

\begin{abstract}
In this paper, we present a synthesis of our fundamental and theoretical research on human system integration and human in-the-loop system for enhancing human performance - especially for technical gestures, in safety critical systems operations such as surgery, astronauts' extra-vehicular activities and aeronautics. Grounding humans-systems integration engineering and design (modelling and simulation) on a formally and experimentally verified theoretical framework, is a necessity to make sure of human in-the-loop system security, safety and reliability. The rise issues concerned with scientific principles of human systems integration and rationale for human in-the-loop systems technical engineering and managerial specific rules.
\end{abstract}

Keywords: human systems integration, human in-the-loop system, performance, security, safety, reliability, theoretical principles, generalized rationale.

\section{Introduction}

A human being, by its biological nature, cannot be reduced to properties of mathematical or physical automaton. Thus, connecting up humans and artefacts is not only a question of technical interaction and interface; it is also a question of integration.

Human systems integration is an umbrella term for several areas of "human factors" research and engineering that include human performance, technology design, and human-interactive systems interaction on six levels, from socio-technical systems to human devices interaction [1]. These are concerned with the integration of human capabilities and performances into the design of complex human-machine systems supporting safe, efficient operations; there is also the question of reliability. Human systems integration is traditionally based on technical and managerial principles [2] [3].

Human systems integration involves augmented human design with the objectives of increasing human capabilities and improving human performance using behavioural technologies [4]. By using wearable interactive systems, made up of virtual environments technologies-like or wearable robotics, many applications offer technical gesture assistance e.g. in aeronautics, human space activities or surgery.

\footnotetext{
Convention CIFRE N³93/2008.
} 
Gesture is a highly integrated neuro-cognitive behaviour, based on the dynamical organization of multiple physiological functions [5] [6]. Assisting gestures and enhancing human skill and performances requires coupling sensorimotor functions and organs with technical systems through artificially generated multimodal interactions. Thus, augmented human design has to integrate human factors anatomy, neurophysiology, behaviour - and assistive cognitive and interactive technologies in a safe and coherent way for extending and enhancing the ecological domain of life and behaviour.

\subsection{Human In-the-Loop System}

The goal of this type of human in-the-loop system design is to create entities that can achieve goals and actions (predetermined) beyond natural human behavioural, physical and intellectual abilities and skills - force, perception action, awareness, decision...

Augmenting cognition and sensorimotor loops with automation and interactive artefacts enhances human capabilities and performance. It is extending both the anatomy of the body and the physiology of human behaviour. Designing augmented human beings by using virtual environment technologies requires integrating both artificial and structural elements and their structural interactions with the anatomy, and artificial multimodal functional interactions with the physiological functions.

Therefore, the scientific and pragmatic questions are: how to best couple and integrate in a coherent way, a biological system with physical and artifactual systems, the less or more immersive interactive and invasive or not artefact, in a behaviourally coherent way by design? How augmented human engineering can anticipate and validate a technical and organizational design? How modelling and assessing such a design?

This paper focuses on one of the main issues for augmented human engineering: integrating the biological user's needs in its methodology for designing humanartefact systems integration requirements and specifications. To take into account biological, anatomical and physiological requirements we need a validated theoretical framework. We propose to ground augmented human engineering on the Chauvet mathematical theory of integrative as a fundamental framework for human system integration and augmented human design. We propose to validate and assess augmented human domain engineering models and prototypes by experimental neurophysiology.

\section{Augmented Human Domain Engineering}

Human-Artefact systems are a special kind of systems of systems. They are made up of two main categories of systems. These two kinds of systems differ in their nature: their fundamental organization, complexity and behaviour. The first category, the traditional one, includes technical or artifactual systems that could be engineered. The second category includes biological systems: the human that could not be engineered. Thus, integrating human and complex technical systems in design is to couple and integrate in a behaviourally coherent way, a biological system (the human) with a 
technical and artifactual system. Augmented human engineering needs to model the human body and its behaviour to test and validate augmented human reliability and human systems integration (HSI).

\subsection{Domain Engineering}

According to system engineering, taking into account user needs in the world of activities and tasks, designing system requirements is to find the system model, its three dimensional organizational dimensions of requirements - structural, functional and dynamical - and its three view plans of system specifications -architecture, behavior and evolution. (Fig.1).

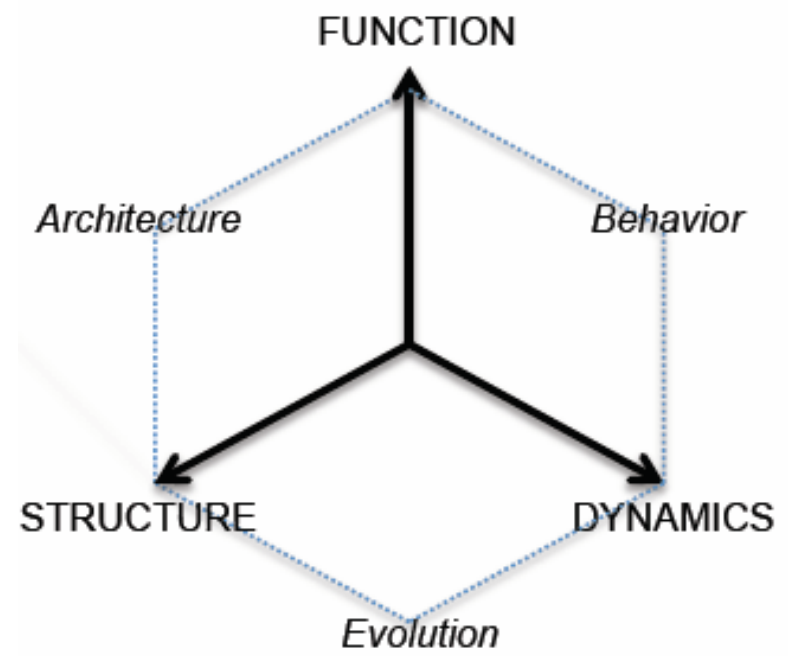

Fig. 1. Grounding human systems integration design is integrating in a total system model, its three dimensional organizational dimensions of requirements - structure, function and dynamics, and its three plans of system specifications - architecture, behavior and evolution, according to fundamental scientific principles. These basic classes of elements of "integrative design" are defined by generalization at the more common levels and domains of hierarchical structural and functional organization of a biological system or an artefactual system.

Thus, system engineering requires both expert skills and validated formal modelling methodologies. To some extent, the main difficulty is to build a system model from a collection of informal and sometimes imprecise, redundant and unstructured descriptions to the domain of expertise. A formal model could be relevant to highlight a hidden structure according to an intended function and its dynamics, or to apply operations or transformation on the system itself.

From domain engineering to requirements, our approach is situated inside Dines Bjoemer's framework [7] [8] [9] based on the triptych: D,S $\rightarrow \mathrm{R}$, where D is the problem domain and where requirements $\mathrm{R}$ are satisfied by the relation $->$, which intends to mean entailment ; so, $\mathrm{S}$ is some model of our system built or expressed 
from $\mathrm{D}$. If that triptych is able to express, in a synthetic manner, a situation related to the problem domain, a system model and the requirements, it remains at a global level and can thus be applied in different problem spaces and instances.

The domain provides a way to express properties and facts of the environment of the system under construction. The system model $\mathrm{S}$ is intended to summarize actions and properties of the system and it is a link between the requirements and the final resulting system. The relation $->$ is conceptualized as a deduction-based relation which can be defined in a formal logical system, and which helps to derive requirements from domain and model. This relation is sometimes called entailment and is used to ground the global framework. When one considers an application, one should define the application domain from the analysis and this may integrate elements of the world. The triptych helps for defining a global framework and offers the possibility to use tools which are useful for assessing the consistent relation between $\mathrm{D}, \mathrm{S}$ and $\mathrm{R}$; because we aim to use proof techniques for ensuring the soundness of the relation.

\subsection{Human System Integration}

The major benefits of using augmented human modelling in design include reducing the need for physical development; reducing design costs by enabling the design team to more rapidly prototype and test a design; avoiding costly design 'fixes' later in the program by considering human factors requirements early in the design process; and improving customer communications at every step of product development by using compelling models and simulations. Thus, designing an artefact consists of organizing a coherent relation between structures and functions in a culture and context of usage [design=structure/function]. Modelling human beings consists of taking into account anatomical and physiological elements in the same model. It is to design functions by organizing a hierarchy of structural elements and their functions [human modelling=physiology (functions)/anatomy (structures)]. Such models should be used to create models of individuals rather than using aggregated summaries of isolated functional or anthropometric variables that are more difficult for designers to use. Therefore augmented human modelling in design requires an integrative approach according to the three necessities we defined for human systems integration [10].

\subsection{Human Systems Integration Domain}

Since technical systems are mathematically grounded and based on physical principles, HITLS needs to be considered in mathematical terms. There are several necessities to make HIS and augmented human reliable [11].

Necessity 1 - Designing a HITLS is to couple two systems from different domains organized and grounded on different principles theory and framework: biological, physical, numerical.

Necessity 2 - HITLS design is a global and integrative model based method ground on Chauvet's Mathematical Theory of Integrative Physiology and domain system engineering. 
Necessity 3 - Modelling augmented human and HSI is to organize the required hierarchically structures and functions and their functional interactions related to dynamics

Consequently, designing an augmented human following human systems integration rationale is to organize hierarchically and dynamically human and artifact coupling. This requires a new domain engineering approach for requirements and specification based on biological user's needs.

\subsection{Augmented Human Engineering}

Dealing with augmented human engineering is being able to situate and limit its domain for specifying the whole system - biological and artefactual integrated system- in accordance with the high-level and global requirements:

D: The ecology of the augmented human: scientific validated principals of augmented human needs;

R: Augmented human teleonomy, augmented human economy and ethics;

S: Biological, Technical and organizational specification of the human-system integration architecture, behavioural performance, stability and reliability.

\section{Augmented Human's Need}

\subsection{Epistemological Needs}

Converging technologies for improving human performances [12], augmented human, need a new epistemological and theoretical approach to the nature of knowledge and cognition considered as an integrated biological, anatomical, and physiological process, based on a hierarchical structural and functional organization. Current models for human-machine interaction or human-machine integration are based on symbolic or computational cognitive sciences and related disciplines. Even though they use experimental and clinical data, they are not yet based on logical, linguistic and computational interpretative conceptual frameworks of human nature, where postulate or axiomatics replace predictive theory. It is essential for the robust modeling and the design of future rules of engineering for HIS, to enhance human capabilities and performance. Augmented human design needs an integrative theory that takes into account the specificity of the biological organization of living systems, according to the principles of physics, and a coherent way to organize and integrate structural and functional artificial elements. Consequently, virtual environments design for augmented human involves a shift from a metaphorical, and scenario based design, grounded on metaphysical models and rules of interaction and cognition, to a predictive science and engineering of interaction and integration. We propose to ground HSI and augmented human design on an integrative theory of the human being and its principles.

\subsection{CHAUVET's Mathematical Theory of Mathematical Physiolgy (MTIP) Needs}

The mathematical theory of integrative physiology, developed by Gilbert Chauvet [14] [15] [16], examines the hierarchical organization of structures (i.e., anatomy) and 
functions (i.e., physiology) of a living system as well as its behaviour. MTIP introduces the principles of a functional hierarchy based on structural organization within spaces limits, functional organization within time limits and structural units that are the anatomical elements in the physical space. It copes with the problem of structural discontinuity by introducing functional interaction, for physiological function coupling, and structural interaction $y$ from structure-source $s$ into structure-sink $S$, as a coupling between the physiological functions supported by these structures.

Unlike interaction in physics, at each level of organization functional interactions are non-symmetrical, leading to directed graph, non local, leading to non local fields, and increase the functional stability of a living system by coupling two hierarchical structural elements. As G. Chauvet said: "we have chosen a possible representation related to hierarchical structural constraints, and which involves specific biological concepts. We also made the important hypothesis that a biological system may be

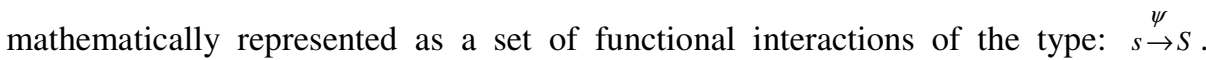
However, the main issue now is to determine whether there exists a cause to the existence of functional interactions, i.e. to the set of triplets $s \stackrel{\psi}{\rightarrow} S$ ? What is the origin of the existence (the identification) of $s, S$ and $y$ that together make a component $\stackrel{\psi}{\rightarrow} S$ of the system? The answer to this issue is the existence of a mathematical principle, the stabilizing auto-association principle or PAAS, a principle that makes of a framework, the MTIP, a veritable theory. The PAAS may be stated as follows: For any triple $(s y S)$, denoted as $\stackrel{\psi}{\rightarrow} S$, where $s$ is the system-source, $S$ the system-sink, and $y$ the functional interaction, the area of stability of the system $\stackrel{\psi}{\rightarrow} S$ is larger than the areas of stability of $s$ and $S$ considered separately. In other words, increasing in

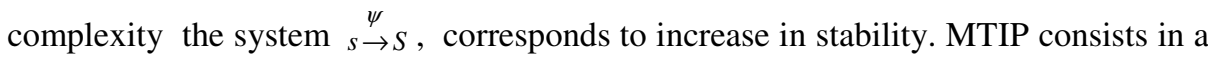
representation (set of non-local interactions $s \stackrel{\psi}{\rightarrow} S$ ), an organizing principle (the PAAS), and a hypothesis (any biological system may be described as a set of functional interactions) that gives rise to two faces of the biological system, the (O-FBS) and the (D-FBS). The first one may be studied using the potential of organization, the second one using the S-Propagator formalism, which describes the dynamics in the structural organization, making an n-level field theory. Both are based on geometrical/topological parameters, and coupled via geometry/topology that may vary with time and space (state variables of the system) during development and adult phases. The structures are defined by the space scale $k$, hence the structural hierarchy, the functions are defined by the time scale $T$, hence the functional hierarchy. Any model built in this theoretical framework will use the same representation, the same basic principle and hypothesis, and consequently will be comparable and able to be coupled with any other on".

\section{Rationale for a Model of Human In-the-Loop System}

Who would even think about separating a living goldfish from its water and its fishbowl! 
As claims by Fass [4], since artifactual systems are mathematically founded and based on physical principles, HSI needs to be thought of in mathematical terms. In addition, there are several main requirements categories to make HIS and human inthe-loop system design, modelling and simulation, safe and efficient. They address the technology - virtual environment-, sensorimotor integration and coherency.

Requirement 1: Virtual Environment is an Artifactual Knowledge based Environment. As an environment, which is partially or totally based on computergenerated sensory inputs, a virtual environment is an artificial multimodal knowledgebased environment. Virtual reality and augmented reality, which are the most well known technologies of virtual environments, are obviously the tools for the augmented human design and the development of human in-the-loop systems. Knowledge is gathered from interactions and dynamics of the individual-environment complex. It is an evolutionary, adaptive and integrative physiological process, which is fundamentally linked to the physiological functions with respect to emotions, memory, perception and action. Thus, designing an artifactual or a virtual environment, a sensorimotor knowledge based environment, consists of making biological individual and artifactual physical system consistent. This requires a neurophysiological approach, both for knowledge modeling and human in-the-loop design.

Requirement 2: Sensori-motor Integration and Motor Control. Humans use multimodal sensori-motor stimuli and synergies for interacting with their environment, either natural or artificial (vision, vestibular stimulus, proprioception, hearing, touch, taste...) [17]. When an individual is in a situation of immersive interaction, wearing head-mounted display and looking at a three-dimensional computer-generated environment, his or her sensorial system is submitted to an unusual pattern of stimuli. This dynamical pattern may largely influence the balance, the posture control, the spatial cognition and the spatial motor control of the individual. Moreover, the coherence between artificial stimulation and natural perceptual input is essential for the perception of the space and the action within. Only when artificial interaction affords physiological processes is coherence achieved.

Requirement 3: Coherence and HIS. If this coherence is absent, perceptual and motor disturbances appear, as well as balance troubles, illusions, vection or vagal reflex. These illusions are solutions built by the brain in response to the inconsistency between outer sensorial stimuli and physiological processes. Therefore, the cognitive and sensorimotor abilities of the person may be disturbed if the design of the artifactual environment does not take into account the constraints imposed by human sensory and motor integrative physiology. The complexity of physiological phenomena arises from the fact that, unlike ordinary physiological systems, the functioning of a biological system depends on the coordinated action of each of the constitutive elements. This is why the designing of a virtual environment as an augmented biotic system, calls for an integrative approach.

Integrative design strictly assumes that each function is a part of a continuum of integrated hierarchical levels of structural organization and functional organization as described above within mathematical theory of integrative physiology (MTIP). Thus, the geometrical organization of the artifactual structure, the physical structure of interfaces and the generated patterns of artificial stimulations, condition the dynamics of hierarchical and functional integration. Functional interactions, which are products or signals emanating from a structural unit acting at a distance on another structural unit, are the fundamental elements of this dynamic. 


\section{Conclusion and Perspective}

By designing a human-artifact system consists in organizing the linkage of multimodal biological structures, sensorimotor elements at the hierarchical level of the living body, with the artificial mechanical or interactive elements of the system, devices and patterns of stimulation. There exists a "transport" of functional interaction in the augmented space of both physiological and artefactual units, and thus a function may be viewed as the final result of a set of functional interactions that are hierarchically and functionally organized between the artificial and biological systems.

Architecture: spatial organization of the structural elements, natural and artificial, coupled by non-local and non-symmetric functional interactions according to PAAS. It is specifying the function(s) of the integrated system. Different organizations specify different architecture and their specific functions:

Behavior: temporal organisation of the patterns of artificial functional interactions, condition and specify the dynamics of augmented sensorimotor loops. It is determining human in-the-loop system behaviour.

Evolution: the spatio-temporal organization of the structural elements and the functional interactions they produce and process specify functional stability of human-artefact system according to an optimum principle -the Chauvet's orgatropy principle, during the life of human in-the-loop system.

Contingent on ecology and economy, architecture, behaviour and evolution as specified, define and limit the life domain of human in-the-loop system.

MTIP is thus applicable to different space and time level of integration in the physical space of the body and the natural or artificial behavioural environment; from molecular level to socio-technical level; from drug design to wearable robotics, and to life and safety critical systems design.

Future works should address questions related to the development of formal models [18], [19] or co-simulation [20] related to human systems integration engineering and design. New questions arise when dealing with deontic or ethical questions that might be handled by human systems integration together with classical formal modelling languages based on deontic or modal languages.

Industrial scientific and ethical challenges rely on designing intelligent and interactive artefactual systems relating machines and human beings. This relationship must be aware of its human nature and its body: it is anatomy and functions. The man-machine interface becomes an integrated continuation of the body between perception-action and sensory and motion organs. By integrating human body and behaviours, the automaton is embodied but this embodiment grounds on the user's body; it enhances capabilities and performances. Safety and reliability rely on aspect of these fundamental necessities.

That is a generalized rationale for guaranteeing the effectiveness of the overall system.

\section{References}

1. Nasa Human System Integration Division, http: / / humanfactors.arc.nasa.gov/index.php

2. Booher, H.R.: Introduction: human systems intégration. In: Booher, H.R. (ed.) Handbook of Human Systems Integration, pp. 1-30 (2003) 
3. Hobbs, A., Adelstein, B., O'Hara, J., Null, C.: Three principles of human-system integration. In: Proceedings of the 8th Australian Aviation Psychology Symposium, Sydney, Australia (April 8-11, 2008)

4. Fass, D.: Rationale for a model of human systems integration: The need of a theoretical framework. Journal of Integrative Neuroscience 5(3), 333-354 (2006)

5. Kelso, J.A.: An Essay on Understanding the Mind. Ecol. Psychol. 20(2), 180-208 (2008)

6. de'Sperati, C., Viviani, P.: The relationship between curvature and velocity in twodimensional smooth pursuit eye movements. J. Neurosci. 17(10), 3932-3945 (1997)

7. Bjorner, D.: Software Engineering 1 Abstraction and Modelling. In: Texts in Theoretical Computer Science. An EATCS Series. Springer, Heidelberg (2006), ISBN: 978-3-54021149-5

8. Bjorner, D.: Software Engineering 2 Specification of Systems and Languages. In: Texts in Theoretical Computer Science. An EATCS Series. Springer, Heidelberg (2006), ISBN: 978-3-540-21150-1

9. Bjorner, D.: Domain Engineering Technology Management, Research and Engineering. COE Research Monograph Series, Vol. 4, JAIST (2006)

10. Fass, D.: Integrative Physiological Design: A Theoretical and Experimental Approach of Human Systems Integration. In: Harris, D. (ed.) HCII 2007 and EPCE 2007. LNCS (LNAI), vol. 4562, pp. 52-61. Springer, Heidelberg (2007)

11. Fass, D., Lieber, R.: Rationale for human modelling in human in the loop systems design. In: $3^{\text {rd }}$ Annual IEEE International Systems Conference, SysCon, Vancouver, pp. 27-30 (2009)

12. Roco, M.C., Brainbridge, W.S.: Converging technologies for improving human performance. National Science Foundation (2003)

13. Chauvet, G.A.: Hierarchical functional organization of formal biological systems: a dynamical approach. I. An increase of complexity by self-association increases the domain of stability of a biological system. Phil Trans Roy Soc London B 339, 425-444 (1993)

14. Chauvet, G.A.: Hierarchical functional organization of formal biological systems: a dynamical approach. II. The concept of non-symmetry leads to a criterion of evolution deduced from an optimum principle of the (O-FBS) sub-system. Phil Trans Roy Soc London B 339, 445-461 (1993)

15. Chauvet, G.A.: Hierarchical functional organization of formal biological systems: a dynamical approach. III. The concept of non-locality leads to a field theory describing the dynamics at each level of organization of the (D-FBS) sub-system. Phil Trans Roy Soc London B 339, 463-481 (1993)

16. Sporns, O., Edelman, G.: Bernstein's dynamic view of the brain: the current problems of modern neurophysiology. Motor Control 2, 283-305 (1988)

17. Cansell, D., Méry, D.: The Event-B Modelling Method: Concepts and Case Studies, in Logics of specification languages. In: Bjørner, D., Henson, M.C. (eds.) Monographs in Theoretical Computer Science, pp. 47-152. Springer, Heidelberg (2008)

18. Mermet, B., Méry, D.: Safe combinations of services using B. In: McDermid, J. (ed.) SAFECOMP 1997 The 16th International Conference on Computer Safety, Reliability and Security. Springer, New York (1997)

19. Leclerc, T., Siebert, J., Chevrier, V., Ciarletta, L., Festor, O.: Multi-Modeling and CoSimulation-based Mobile Ubiquitous Protocols and Services Development and Assessment. In: 7th International ICST Conference on Mobile and Ubiquitous Systems (2010) 